\title{
BMJ Open A randomised controlled trial of an intervention delivered by app instant messaging to increase the acceptability of effective contraception among young people in Tajikistan: study protocol
}

\author{
Ona McCarthy, ${ }^{1}$ Baptiste Leurent, ${ }^{2}$ Phil Edwards, ${ }^{1}$ Ravshan Tokhirov, ${ }^{3}$ \\ Caroline Free $^{1}$
}

To cite: McCarthy 0 ,

Leurent B, Edwards P, et al. A randomised controlled trial of an intervention delivered by app instant messaging to increase the acceptability of effective contraception among young people in Tajikistan: study protocol. BMJ Open 2017;7:e017606. doi:10.1136/ bmjopen-2017-017606

- Prepublication history and additional material for this paper are available online. To view please visit the journal (http:// dx.doi.org/10.1136/bmjopen2017-017606).

Received 2 May 2017

Revised 29 June 2017 Accepted 21 July 2017

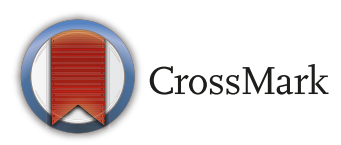

${ }^{1}$ Department of Population Health, The London School of Hygiene and Tropical Medicine, London, UK

${ }^{2}$ Department of Infectious Disease Epidemiology, London School of Hygiene and Tropical Medicine, London, UK

${ }^{3}$ Tajik Family Planning Association, Dushanbe, Tajikistan

Correspondence to Ona McCarthy; ona.mccarthy@Ishtm.ac.uk

\section{ABSTRACT}

Introduction Women in lower income countries experience unintended pregnancies at a higher rate compared with women in higher income countries. Unintended pregnancy is associated with numerous poorer health outcomes for both women and their children. In Tajikistan, an estimated $26 \%$ of married individuals aged 15-24 years have an unmet need for contraception. The strong cultural value placed on childbearing and oppositional attitudes towards contraception are major barriers to contraceptive uptake in the country. Mobile phone ownership is widespread in Tajikistan. The option of receiving reproductive health support on your personal phone may be an appealing alternative to attending a clinic, particularly for young people. The London School of Hygiene \& Tropical Medicine and the Tajik Family Planning Association have partnered to develop and evaluate a contraceptive behavioural intervention delivered by mobile phone. The intervention was developed in 2015-2016 guided by behavioural science. It consists of short instant messages sent through an app over 4 months, contains information about contraception and behaviour change methods.

Methods and analysis This randomised controlled trial is designed to evaluate the effect of the intervention on self-reported acceptability of effective contraception at 4 months. 570 men and women aged 16-24 years will be allocated with a ratio of 1:1 to receive the intervention messages or the control messages about trial participation. The messages will be sent through the Tajik Family Planning Association's 'healthy lifestyles' app, which contains basic information about contraception. Ethics and dissemination The trial was granted ethical approval by the London School of Hygiene \& Tropical Medicine Interventions Research Ethics Committee on 16 May 2016 and by the Tajik National Scientific and Research Centre on Paediatrics and Child Surgery on 15 April 2016. The results of the trial will be submitted for publication in peer-reviewed academic journals and disseminated to study stakeholders.

Trial registration number Clinicaltrial.gov NCT02905513. Date of registration 14 September 2016.

WHO trial registration dataset http://apps.who.int/ trialsearch/Trial2.aspx?TriallD=NCT02905513
Strengths and limitations of this study

- The intervention was developed using an established approach grounded in behavioural science.

- Participants must own a personal Android phone to receive the intervention, which means that the sample may not represent young people from a broad socioeconomic spread.

- The randomised trial design will allow us to conclude that an observed effect was due to the intervention.

- This is the first trial to evaluate an intervention for contraception delivered by mobile phone for young people in Tajikistan.

\section{INTRODUCTION}

Globally in 2012, an estimated 85 million pregnancies (approximately $40 \%$ of all pregnancies) were unintended. ${ }^{1}$ Women in lower income countries experience more unintended pregnancies than in higher income countries, with a rate of 54 per 1000 women compared with 44 per 1000 women, respectively. ${ }^{1}$ Unintended pregnancy is associated with numerous poorer health and outcomes for both women and their children ${ }^{2}$ including decreased psychological well-being ${ }^{3-11}$ and delay in initiating antenatal care. ${ }^{511-15}$ Children born of unintended pregnancies are at a higher risk of low birth weight and preterm birth. ${ }^{16}{ }^{17}$ A woman has an unmet need for modern contraception if they want to avoid a pregnancy but currently use no method or a traditional method. ${ }^{18}$ Fulfilling unmet need for contraception is essential in decreasing unintended pregnancy; however, in 2014, an estimated 225 million women in low-income and middle-income countries had an unmet need for modern contraception. ${ }^{18}$

The civil war that followed Tajikistan's independence from the Soviet Union in 1991 
had devastating effects on the Tajik economy and health system. ${ }^{19}{ }^{20}$ While economic hardship continues, fundamental reforms to the healthcare system have instigated progressive initiatives, such as the adoption of the Strategic Plan for Reproductive Health in 2004. ${ }^{21}$ With regard to family planning data, the Tajikistan Demographic and Health Survey (DHS) 2012 is the most reliable source at present. ${ }^{21}$ Effective contraception methods are those with a less than $10 \%$ typical use failure rate at 12 months. ${ }^{22-24}$ The effective methods available in Tajikistan are oral contraceptive pills, intrauterine devices (IUDs), injectables and implants. While there is some concern about the suitability of the progestogen-only injection for adolescents, adolescents are eligible to use the same methods as adults; age alone is not a contraindication for use. ${ }^{25}$ Despite the availability of the effective methods in Tajikistan, it is estimated that $26 \%$ of married individuals aged 15-24 years have an unmet need for contraception. Total unmet need peaks at age 25-29, reflecting the cultural norm of early family completion.

The 2012 DHS survey estimated that around 23\% of all married women and $8 \%$ of married individuals aged 15-24years report using an effective method, the IUD being the most popular. ${ }^{21}$ Eighty-three per cent of induced abortions (all age groups) occur in women not using any method. ${ }^{21}$ The total wanted fertility rate is reported to be 3.3 compared with the actual of 3.8 , indicating that woman have 0.5 children more than desired. ${ }^{21}$ Oppositional attitudes towards contraception are a barrier to use in Tajikistan; $36 \%$ of women with an unmet need cite their own opposition and 13\% cite their partner's opposition as the reason for not using contraception. ${ }^{26}$ Other reasons married women with an unmet need gave for not using contraception were infrequent/no sex (28\%) and side effects/health risks/inconvenience (15\%). ${ }^{26}$

Health interventions delivered by mobile phone are increasingly popular. ${ }^{27-37}$ With sensitive topics such as reproductive health, the ability to receive information on a personal mobile phone may be an appealing alternative to attend a clinic. While there is some evidence from high-income countries that mobile phone-based interventions can increase contraceptive-related behaviours ${ }^{38-40}$ and knowledge, ${ }^{41}$ none of the trials evaluating these interventions had a low risk of bias ${ }^{42}$ according to the Cochrane Collaboration's tool for assessing risk of bias in randomised controlled trials. ${ }^{43}$ The tool assesses risk over multiple domains of bias, that is, selection, performance, detection, attrition, reporting and other bias. To the best of our knowledge, there is only one trial conducted in a non-high income country (Cambodia). This trial found an effect of postabortion voice messaging with telephone counselling support on effective contraceptive use. ${ }^{44}$

The London School of Hygiene and Tropical Medicine (LSHTM) and the Tajik Family Planning Association (TFPA), a member association of the International Planned Parenthood Federation (IPPF), are collaborating to evaluate an intervention delivered by mobile phone for young men and women in Tajikistan to increase the acceptability of effective contraception. In Tajikistan in 2015, there were 98.6 mobile phone subscriptions per 100 people ${ }^{45}$ with $64 \%$ owning a smartphone. ${ }^{46}$ The intervention was developed in 2015-2016 guided by an evidencebased approach grounded in behavioural science. ${ }^{47}$ The approach involved consultation with the target group to identify attitudinal barriers to contraceptive use and an iterative process of writing intervention content, testing with the target group and refining. This development work indicated that short messages delivered by mobile phone could be an acceptable way to provide contraceptive support to young people.

The aim of this publication is to present the protocol for the evaluation of the intervention by randomised controlled trial. The trial is designed to evaluate the effect of the intervention on young Tajik people's self-reported acceptability of the effective contraception methods available in Tajikistan. To the best of our knowledge, this will be the first trial evaluating an intervention delivered by mobile phone designed to increase the acceptability of effective contraception in Tajikistan. ${ }^{42}$

\section{METHODS AND ANALYSIS \\ Design}

This study is a parallel group, individually randomised superiority trial with a 1:1 allocation ratio evaluating the effect of an intervention delivered by mobile phone application (app). Participants randomised to the intervention arm will have access to the app and will receive the intervention instant messages. Participants randomised to the control arm will have access to the app and receive control instant messages about trial participation.

\section{Eligibility criteria}

Women and men aged 16-24 years, who own a personal Android mobile phone and live in Tajikistan, can provide informed consent and can read Tajik or Russian will be eligible to take part. Participants must be willing to receive messages about contraception on their mobile phone.

\section{Recruitment}

The trial will be promoted through the distribution of flyers through TFPA's volunteers and youth partner organisation, TFPA's website and social media sites. Potential participants will be provided the link to the enrolment pages of the secure online trial database and randomisation system, where they will read the participant information sheet (online supplementary file 1) and provide informed consent (online supplementary file 2). If they do not have adequate internet access, youth organisation volunteers will provide it. Participants will also have the option of completing the paper-based version of the consent form.

To maximise the chance of recruiting to target, LSHTM conducted a pretrial training in Dushanbe to train local staff on all recruitment procedures. The training included a discussion about the practicalities of recruitment with a 


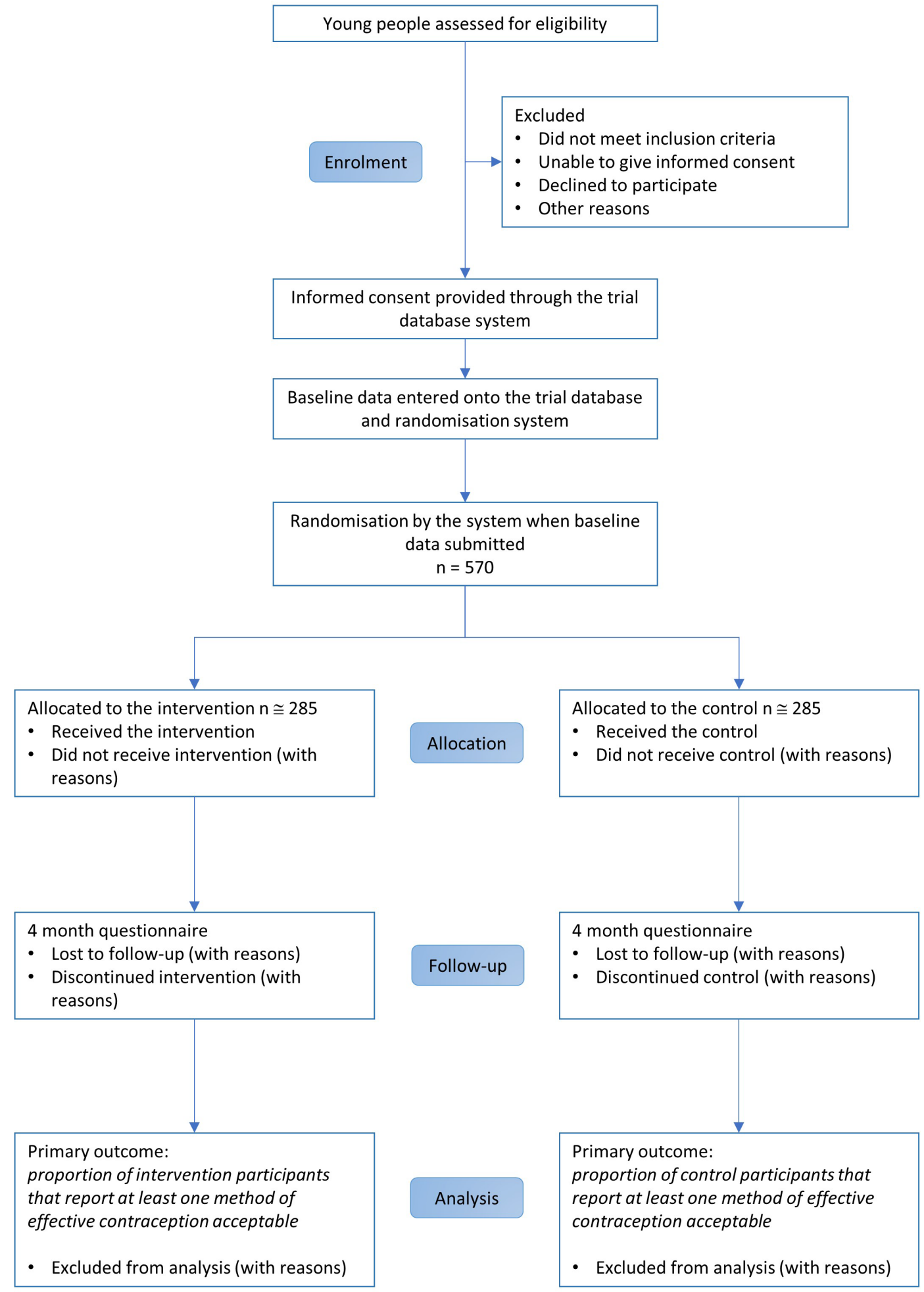

Figure 1 CONSORT diagram.

view to developing the most appropriate strategies (ie, the distribution of flyers, an advertisement on TFPA's website and social media sites).

We will report the number of people assessed for eligibility, number of people excluded before randomisation, number of participants randomised, number of people allocated to the intervention and number of people who completed the follow-up and analyse (figure 1).

\section{Intervention}

The intervention is informed by the Integrated Behavioural Mode ${ }^{48}$ and consists of short mobile phone app instant messages that provide contraceptive support delivered over 4 months sent through TFPA's 'healthy lifestyles' app. The intervention messages provide information about contraception, target beliefs identified in the development phase that influence contraceptive use (eg, 


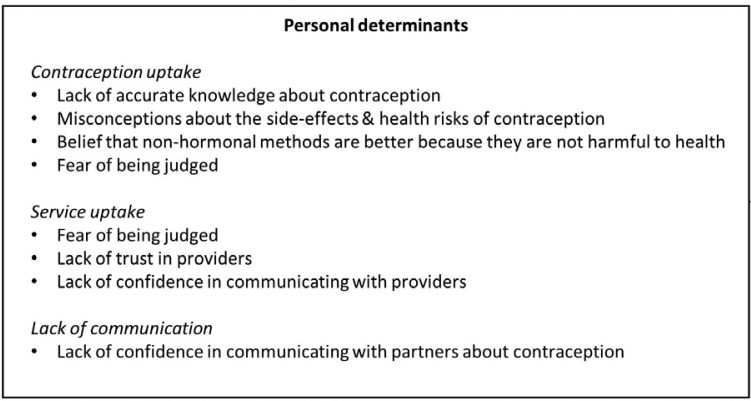

Figure 2 Logic model of the problem.

misconceptions about the side effects and health risks of contraception and belief that non-hormonal methods are better because they are not harmful to health) and aim to support young women in believing that they can influence their reproductive health (see figure 2 for the logic model of the problem). The intervention provides accurate information about contraception and contains the following behaviour change methods, ${ }^{49}$ adapted for delivery by mobile phone: belief selection, facilitation, anticipated regret, guided practice, verbal persuasion, tailoring, cultural similarity, arguments, shifting perspective and goal setting. The app itself contains basic information about contraception, how to have a 'healthy lifestyle', youth friendly service point locations and contains no behaviour change methods. The app and the intervention messages are available in Tajik or Russian, according to participants' preference, which is indicated at enrolment.

The messages are tailored according to marital status and gender, resulting in four sets of intervention messages: (1) female-married, (2) female-not married, (3) male-married and (4) male-not married. Most of the messages in the four sets overlap, with minor tailoring so that the messages are relevant to marital status and gender. Marital status is used as a proxy for sexual activity because the target group and TFPA considered it inappropriate to ask directly about sexual activity. Based on the development work with the target group, participants receive 0-3 messages per day (135 messages for female-not married, 155 messages for female-married, 135 messages for male-not married and 146 messages for male-married) for 120 days. Included in the messages that intervention recipients receive are seven control messages about the importance of their participation and reminding them to contact the project coordinator if they change their number.

The message sets start with 6-7 days of messages (11 female-married, 12 female-not married, 12 male-married and 13 male-not married) with general information about the study, such as what they will receive over the next 120 days, how to stop the messages, how to choose specific times to receive the messages, who to contact if they change their number, how to keep the messages private and information about who to call if they feel unsafe as a result of someone reading the messages (women only). On the final 2-3 days, the message sets include four messages that indicate that the messages have ended, provide a link to the database to complete the follow-up questionnaire, reassurance that the information that they provide is confidential and a final message stating that their participation is helping to determine the best ways to provide reproductive health services in Tajikistan.

Details regarding the development of the intervention and intervention description will be reported in a forthcoming publication.

\section{CONTROL}

Participants allocated to the control group will have access to the same app pages as the intervention group. Control participants will also receive 16 messages about trial participation over 120 days. The first 4 days include six messages that introduce the study, provide information about what they will receive over the next 120 days, how to stop the messages and who to contact if they change their number. They will then receive two messages a month for 3 months-one about the importance of their participation and one reminding them to contact the project coordinator if they change their number. On day 105 , they will receive one message about the importance of their participation. On day 120, participants will receive three messages that provide information on how to complete the follow-up questionnaire, reassurance that the information that they provide is confidential and a final message stating that their participation is helping to determine the best ways to provide reproductive health services in Tajikistan.

All participants will receive usual care (the normal care that a young person would receive if they attended a service in Tajikistan) and will be free to seek any other support, whether existing or new. TFPA's app is part of 'usual care' for the purpose of the trial and will be 'new usual care' after the trial.

\section{OUTCOMES}

\section{Primary outcome}

The primary outcome is the proportion of participants reporting that at least one method of effective contraception is acceptable at 4 months postrandomisation. In the absence of an existing validated measure of acceptability that was appropriate for this context, the primary 
outcome measure was constructed based on guidelines for measuring IBM constructs ${ }^{48} 51$ and tested for face validity with the target group. The acceptability of each method is measured by the following stems: 'Using the [method] ...causes infertility, ...causes unwanted side effects, ... is easy, ... is a good way to prevent pregnancy and I would recommend the [method] to a friend'. The IUD and implant include an additional stem: 'The [method] insertion would not be a problem for me. The response options for each scale are: strongly disagree, disagree, not sure, agree, strongly agree and I do not know what the [method] is. A method is acceptable if participants report 'agree' or 'strongly agree' for all scales except for '...causes infertility' and '...causes unwanted side effects' stems, for which 'disagree' or 'strongly disagree' denotes acceptability (items 1-22 in online supplementary file 3 and items 4-25 in online supplementary file 4).

\section{Secondary outcomes}

Secondary outcomes are: the proportion reporting current use (or partner's use) of effective contraception (use of effective contraception); the proportion reporting that each method of effective contraceptive method is acceptable (acceptability of individual methods); the proportion reporting use (or partner's use) of effective contraception at any time during the 4 months (discontinuation); the proportion reporting attending a sexual health service during the 4 months (service uptake); the proportion reporting that they became pregnant and did not want a pregnancy during the study (unintended pregnancy); and the proportion reporting having (or partner having) an abortion during the study (induced abortion).

\section{Process outcomes}

The process outcomes are: knowledge of effective contraception; perceived norms in relation to using and communicating with partners about contraception; personal agency in using (women only) and communicating with partners about contraception; and intention to use effective contraception (women only) and intervention dose received.

\section{DATA COLLECTION}

Data will be collected at baseline and at 4 months postrandomisation using questionnaires, which we tested for face validity with 27 people from the target group. We asked people to comment on the length of the questionnaires, the comprehensibility of the questions, the meaning of the scales and suggestions for improvement. All data will be entered onto the trial database system, which is on LSHTM's secure server. At both time points, participants can either fill out a paper-based version of the questionnaire at the recruitment site, provide the data over the phone with research staff or enter data directly onto the online system, according to their preference. If participants provide their questionnaire data by paper or over the telephone, research staff will enter these data onto the system.

\section{Baseline data collected}

At baseline we will measure the primary outcome and collect the following personal and demographic data: full name, mobile phone number, email address, date of birth, gender, marital status, number of children, ethnicity, occupation, education level, current pregnancy intention, current method and how they found out about the study (online supplementary file 3 ).

\section{Follow-up data collected}

At 4 months, we will measure the primary, secondary and process outcomes and collect the following data: if participants report using an effective method, where they obtained it, current pregnancy intention, whether they knew someone else that took part in the study and, if so, if they read each other's messages (contamination), if they have experienced physical violence since being in the study and if anything good or bad happened as a result of receiving the messages (online supplementary file 4$)$.

If participants do not complete the questionnaire themselves, local research staff will contact them to collect their data. For participants that report use of effective contraception at follow-up, local research staff will attempt to locate the service records to objectively verify use.

\section{Methods to maximise follow-up response}

The pretrial training also included training in follow-up procedures. It emphasised the importance of ensuring that participants understand that participation involves completing a 4-month questionnaire and to potentially receiving daily messages about contraception for 4 months. The control messages, also sent to participants allocated to the intervention, are an effort to keep participants engaged. Staff will contact non-responders up to three times for their follow-up data. Follow-up will end 6 months after the last participant has been randomised or after staff has attempted to contact all non-responders three times, whichever comes first.

See figure 3 for the schedule of enrolment, interventions and assessments.

\section{Allocation and protecting against bias}

Randomisation will occur immediately after baseline data is submitted on the trial database and randomisation system. The allocation sequence is generated by the remote computer-based randomisation software, ensuring that investigators are unaware of allocation before it occurs. Due to the nature of the intervention, participants will be aware of the allocation soon after they start receiving the messages. Local research staff collecting outcome data will not be made aware of allocation unless this is revealed to them by the participant. Researchers that analyse the data will be masked to treatment allocation. 


\begin{tabular}{|c|c|c|c|c|c|c|c|}
\hline \multirow[b]{3}{*}{ TIMEPOINT } & \multicolumn{7}{|c|}{ STUDY PERIOD } \\
\hline & \multirow{2}{*}{$\frac{\text { Enrolment }}{0}$} & \multirow{2}{*}{$\frac{\text { Allocation }}{0}$} & \multicolumn{4}{|c|}{ Post-allocation } & \multirow{2}{*}{$\begin{array}{c}\text { Close-out } \\
6 \text { months after last } \\
\text { participant randomised }\end{array}$} \\
\hline & & & $\begin{array}{c}\text { Month } \\
1\end{array}$ & $\begin{array}{c}\text { Month } \\
2\end{array}$ & $\begin{array}{c}\text { Month } \\
3\end{array}$ & $\begin{array}{c}\text { Month } \\
4\end{array}$ & \\
\hline Eligibility screen & $\sqrt{ }$ & & & & & & \\
\hline Informed consent & $\sqrt{ }$ & & & & & & \\
\hline Baseline data completion & $\sqrt{ }$ & & & & & & \\
\hline Baseline data entry & $\sqrt{ }$ & & & & & & \\
\hline Allocation & & $\sqrt{ }$ & & & & & \\
\hline $\begin{array}{l}\text { Contraceptive app instant messages } \\
\text { (0-3 messages per day for } 120 \text { days) }\end{array}$ & & & & & & & \\
\hline $\begin{array}{r}\text { App instant messages not about contraception } \\
\text { (16 messages over } 120 \text { days) }\end{array}$ & & & & & & $\longrightarrow$ & \\
\hline \multirow{2}{*}{$\begin{array}{rr}\text { ASSESSMENTS } & \text { Baseline data: } \\
\text { primary outcome, personal and demographic data } & \text { Follow-up data: } \\
& \text { primary, secondary and process outcomes }\end{array}$} & $\sqrt{ }$ & & & & & & \\
\hline & & & & & & $\sqrt{ }$ & $\sqrt{ }$ \\
\hline
\end{tabular}

*Plus: if participants report using an effective method, where they obtained it; current pregnancy intention; whether they knew someone else that took part in the study and if so, if they read each other's messages (contamination); if they have experienced physical violence since being in the study and if anything good or bad happened as a result of receiving the messages

Figure 3 Schedule of enrolment, interventions and assessments.

\section{Intervention delivery}

After participant baseline data has been entered, a confirmation of enrolment screen will provide instructions on how to install the app. When participants install the app, they will be prompted to enter the mobile phone number they entered on the baseline questionnaire. The trial database and randomisation system will then send the local app platform the following information: gender, marital status, language preference, allocation and date of enrolment. Participants will then have access to the app and will receive either the control or intervention messages, according to their allocation. Within the app, participants can choose when they want to receive the messages and they can also stop the messages. If participants install the app after 13:00, they will receive the first message the following day.

\section{Sample size}

The trial is powered to detect a $15 \%$ increase in acceptability of effective contraception in the intervention group compared with the control group. Other studies have found smaller increases in behaviour with similar interventions, for example, Castaño et $_{\text {al. }}{ }^{39}$ Because attitudinal change is likely to be easier to achieve than behavioural change, we decided to power the trial to detect a larger difference. Four hundred and fifty-four participants will allow for $90 \%$ power to detect a $15 \%$ absolute increase in acceptability, assuming $50 \%$ acceptability in the control group (ie, $50 \%$ in the control vs $65 \%$ in the intervention, an OR of 1.86). Fifty per cent baseline acceptability is used in the absence of published data on acceptability in this context. If the actual baseline acceptability is higher or lower than $50 \%$, the trial is still sufficiently powered to detect an absolute difference of $15 \%$. For example, if the proportion in the control arm is $75 \%$, there will be $90 \%$ power to detect an absolute difference of $12 \%$ (corresponding to $87 \%$ acceptability in the intervention group and an OR of 2.23). Allowing for $20 \%$ loss to follow-up, 570 people will be randomised.

\section{DATA MANAGEMENT}

We did not convene a Data Monitoring and Ethics Committee as the intervention provides support and is unlikely to produce adverse effects. We have convened a Trial Steering Committee, and they have agreed to take on the monitoring of ethical aspects of the trial. The trial sponsor may audit the trial according to their own risk assessment and schedule.

Personal details entered onto the trial database and randomisation system will be stored on LSHTM's secure server. Personally identifiable information exported from the database will be stored separately from anonymised research data. Participants' mobile phone numbers, but no other personal details, will be stored in the local platform that sends the messages through the app. Any signed paper consent forms and questionnaires will be kept in a data enclave at TFPA. All data arising from the study will be kept confidential and only accessible to researchers directly involved in it. Personally identifiable data will not be kept longer than necessary and will be deleted within 3 months following study completion. We will retain primary research data for 10 years following study completion.

\section{ANALYSES}

\section{General statistical considerations}

The analysis of the data will follow the plan specified below. There will be no interim analyses and therefore no stopping rules. All analyses will be according to randomised arm, and only participants with complete outcome data 
will be included in the primary analysis (a complete case analysis). All statistical tests will be two sided. All effect estimates will be reported with a 95\% CI and associated $\mathrm{p}$ value. Statistical significance will be considered at the 5\% level. Analyses will be conducted using Stata 15.

\section{Loss to follow-up}

To investigate whether loss to follow-up differs by arm, we will report this descriptively and use a $\chi^{2}$ test. We will use logistic regression to compare baseline characteristics of participants that completed 4-month follow-up against participants that did not. We will report predictors of loss to follow-up and investigate whether the effect of these differs by arm by testing for an interaction.

\section{Assumptions about missing data}

As we are not aware of similar trials, it is not possible to investigate the pattern of missing data. The complete case analysis assumes that missing data for participants that did not complete follow-up are similar to data from participants that completed follow-up, conditionally based on baseline covariates included in the analysis model (ie, that data is missing at random) ${ }^{52}$ If participants that complete follow-up are more likely to find an effective method acceptable compared with those that are lost to follow-up, the observed proportion may overestimate acceptability. ${ }^{52}$

\section{Missing covariates}

The database requires all items on the baseline questionnaire to be submitted to randomise. Therefore, there will be no missing baseline covariates.

\section{Primary analysis}

Descriptive analysis

We will report a flow diagram of trial participation, as recommended in the CONSORT guidelines. ${ }^{53}$ We will report the baseline characteristics by treatment arm. We will also explore the baseline factors associated with retention (see above).

\section{Analysis of the primary outcome}

The primary outcome is binary, and we will compare the crude proportion reporting at least one method is acceptable in each group. We will estimate the difference between the groups using logistic regression and will report the OR along with the $95 \% \mathrm{CI}$ and $\mathrm{p}$ value for evidence against the absence of intervention effect from the model. The primary analysis regression will be adjusted for baseline covariates likely to be associated with the outcome in order to improve the efficiency of the analysis and avoid chance imbalances. ${ }^{54}$ These prespecified covariates that we will adjust for are: use (using effective contraception/ not using effective contraception); pregnancy intention (wants to avoid/other); gender (female/male), age (16-19/20-24 years); number of children $(0 / 1+)$; highest education level completed (university/other) and acceptability of effective contraception at baseline (at least one method acceptable/no methods acceptable). We will also report the crude OR between arms.

\section{Analysis of the secondary outcomes}

The analysis of the secondary outcomes will be the similar to the analysis of the primary outcome. We will estimate the difference between the groups using logistic regression, report ORs with 95\% CIs and $\mathrm{p}$ values. All regressions will be adjusted for the prespecified covariates as above (although with the acceptability of individual methods, the outcome at baseline will replace acceptability of effective contraception).

\section{Analysis of the process outcomes}

The process outcomes perceived norms, personal agency and intention are comprised of ordinal scales. Each scale will be analysed individually using ordered logistic regression to estimate proportional ORs. For knowledge, each correct answer will receive one point. The points will be summed, and an overall score will be produced. We will use linear regression to test for a difference in mean scores between the arms.

To assess the 'dose' of the intervention that the intervention participants received, we will analyse the number of messages that participants reported to have read (all, most, some and none) and whether they stopped the messages. This will be reported descriptively.

\section{Additional analyses}

Sensitivity analyses

We will conduct sensitivity analyses regarding the missing data. In the first sensitivity analysis, we will consider that data are not missing at random and that participants lost to follow-up did not find at least one method acceptable. In the second, we will adjust for the main baseline predictors of missingness. Both sensitivity analyses will be adjusted for the pre-specified covariates as above.

\section{Subgroup analysis}

Recognising that the trial is not powered to detect effect differences in subgroups, we will conduct exploratory subgroup analyses for the primary outcome to determine if the intervention effect varies by baseline characteristics. The prespecified subgroups are: gender (female/male); age (split at the median); marital status (married/not married); number of children $(0 / 1+)$; ethnicity (Tajik/ other); occupation (in education/other); highest education level completed (university/other) and pregnancy intention (wants to avoid/other). Within the prespecified subgroups, we will assess heterogeneity of treatment effect with a test for interaction. ${ }^{55-59}$ Interaction test $p$ values will be presented but will be interpreted with caution, due to the exploratory nature, the multiple tests performed and the low power of the interaction test. We will estimate ORs along with 95\% CIs for each subgroup without $p$ values. As this is an exploratory analysis of potentially influential characteristics that are not justified a priori, we will not hypothesise effect directions.

\section{Contamination}

To assess the potential for contamination, we will report the proportion of control group participants that read 
another participant's messages and the proportion of intervention participants whose messages were read by another participant.

\section{Participants' rights and safety}

Participants will have the right to withdraw at any time during their involvement, without having to give a reason. Participants can withdraw by contacting the project coordinator. Acting on participants' requests to withdraw from the trial, participants' status will be changed to 'withdrawn', and the person will be excluded from the list of participants that are due to follow-up. Participants' participation and personal identifiable data will remain confidential and research data will be anonymised.

In the development phase, we explored young people's views on confidentiality about receiving messages on their mobile phone. While the large majority of participants reported that they were not concerned about receiving messages about contraception on their phone, it is possible that some participants will want to keep the messages confidential from certain people (eg, partner and parents) and that these people might view the messages. The messages remind participants that they can delete the messages and provide instructions on how to keep the messages private. Towards the beginning of the intervention, a message provides female participants with information on support services that they can contact if they feel unsafe as a consequence of the messages being read. (This information will not be provided for male participants as TFPA advised that male participants not feeling safe as a consequence of the messages being read is not culturally realistic.) We will review physical violence during participants' involvement in the trial reported on the follow-up questionnaire.

\section{DISCUSSION}

The results of this trial will provide evidence for the effect of the intervention on young Tajik women and men's attitudes towards effective contraception. The analysis of the secondary and process outcomes may provide evidence for the effect of the intervention on use of effective contraception, attitudes towards the individual effective methods, service use, unintended pregnancy, induced abortion and on the psychological processes that may have been altered by the intervention.

As the intervention will be delivered through TFPA's Android app, participants are required to own a personal Android mobile phone. While the formative work indicated that the majority of young people in Tajikistan did own a personal Android mobile phone, those who do not own a smartphone may be those who are less likely to find at least one method of effective contraception acceptable. However, considering the rapid increase in smartphone ownership, it is reasonable to assume that ownership will be an option for a greater proportion of young people across different socioeconomic communities in the coming years.
The trial is assessing the effect of sending instant messages containing behaviour change methods in addition to the app; it is not assessing the effect of the app itself. It is possible that the app, which provides basic information about contraception, could increase the acceptability of effective contraception. If the app itself is very effective, the added benefit of the instant messages will be lower.

If the trial demonstrates that the intervention increases the acceptability of effective contraception in Tajikistan, the results could inform the design of a trial to evaluate the effect of the intervention on unintended pregnancy.

\section{PROTOCOL AMENDMENTS}

Any important changes to the protocol will be submitted to the LSHTM Interventions Research Ethics Committee as an amendment. Trial documentation will be updated accordingly and will be implemented once the Committee has approved the changes. OLM will communicate any changes relevant to local staff.

\section{DISSEMINATION}

The research results will be cowritten by LSHTM and TFPA and submitted for publication in peer-reviewed academic journals. We will adhere to the International Committee of Medical Journal Editors authorship criteria. We will disseminate findings to all the study stakeholders.

Acknowledgements The authors would like to thank Marieka Vandewiele and Sarah Standaert from the IPPF European Network and Salokhiddin Saibov from TFPA for facilitating decisions regarding the design of the trial though our regular planning meetings and Alison McKinley from IPPF for her support over the course of the project.

Contributors $\mathrm{OM}$ designed and managed the trial, developed the trial materials and wrote the manuscript. RT contributed to discussions and decisions regarding the design of the trial, assisted in the development of the trial material and facilitated trial implementation. RT also took overall local responsibility for the trial. $\mathrm{BL}$ and PE provided advice regarding the statistical analysis. CF provided guidance regarding the trial design and implementation. All authors revised the work, approved the version to be published and agree to be accountable for all aspects of the work.

Funding The trial is supported by the International Planned Parenthood Federation (IPPF) Innovation Programme. IPPF had some influence over the study design (MV and SS) but will have no involvement in the data collection or analysis.

\section{Competing interests None declared.}

Patient consent Detail has been removed from this case description/these case descriptions to ensure anonymity. The editors and reviewers have seen the detailed information available and are satisfied that the information backs up the case the authors are making.

Ethics approval The trial was granted ethical approval by the LSHTM Interventions Research Ethics Committee on 16 May 2016 and by the Tajik National Scientific and Research Centre on Paediatrics and Child Surgery under the Ministry of Health on 15 April 2016.

Provenance and peer review Not commissioned; externally peer reviewed.

Data sharing statement The datasets used and analysed during the current study are available from the corresponding author on reasonable request.

Open Access This is an Open Access article distributed in accordance with the Creative Commons Attribution Non Commercial (CC BY-NC 4.0) license, which permits others to distribute, remix, adapt, build upon this work non-commercially, and license their derivative works on different terms, provided the original work is 
properly cited and the use is non-commercial. See: http://creativecommons.org/ licenses/by-nc/4.0/

(c) Article author(s) (or their employer(s) unless otherwise stated in the text of the article) 2017. All rights reserved. No commercial use is permitted unless otherwise expressly granted.

\section{REFERENCES}

1. Sedgh G, Singh S, Hussain R. Intended and unintended pregnancies worldwide in 2012 and recent trends. Stud Fam Plann 2014;45:301-14.

2. Brown SS, Eisenberg L, eds. The Best Intentions: Unintended Pregnancy and the Well-Being of Children and Families. Washington D.C: National Academies Press, 1995.

3. Hardee K, Eggleston E, Wong EL, et al. Unintended pregnancy and women's psychological well-being in Indonesia. J Biosoc Sci 2004;36:617-26.

4. Khajehpour M, Simbar M, Jannesari S, et al. Health status of women with intended and unintended pregnancies. Public Health 2013;127:58-64.

5. Gipson JD, Koenig MA, Hindin MJ. The effects of unintended pregnancy on infant, child, and parental health: a review of the literature. Stud Fam Plann 2008;39:18-38.

6. Najman JM, Morrison J, Williams G, et al. The mental health of women 6 months after they give birth to an unwanted baby: a longitudinal study. Soc Sci Med 1991;32:241-7.

7. Barber JS, Axinn WG, Thornton A. Unwanted childbearing, health, and mother-child relationships. J Health Soc Behav 1999;40:231-57.

8. Nakku JE, Nakasi G, Mirembe F. Postpartum major depression at six weeks in primary health care: prevalence and associated factors. Afr Health Sci 2006;6:207-14.

9. Lau Y, Keung DW. Correlates of depressive symptomatology during the second trimester of pregnancy among Hong Kong Chinese. Soc Sci Med 2007;64:1802-11.

10. Orr ST, Miller CA. Unintended pregnancy and the psychosocial wellbeing of pregnant women. Womens Health Issues 1997;7:38-46.

11. Cheng D, Schwarz EB, Douglas E, et al. Unintended pregnancy and associated maternal preconception, prenatal and postpartum behaviors. Contraception 2009;79:194-8.

12. Eggleston E. Unintended pregnancy and women's use of prenatal care in Ecuador. Soc Sci Med 2000;51:1011-8.

13. Marston C, Cleland J. Do unintended pregnancies carried to term lead to adverse outcomes for mother and child? An assessment in five developing countries. Popul Stud 2003;57:77-93.

14. Kost K, Landry DJ, Darroch JE. Predicting maternal behaviors during pregnancy: does intention status matter? Fam Plann Perspect 1998;30:79-88

15. Magadi MA, Madise NJ, Rodrigues RN. Frequency and timing of antenatal care in Kenya: explaining the variations between women of different communities. Soc Sci Med 2000;51:551-61.

16. Shah PS, Balkhair T, Ohlsson A, et al. Intention to become pregnant and low birth weight and preterm birth: a systematic review. Matern Child Health J 2011;15:205-16.

17. Mohllajee AP, Curtis KM, Morrow B, et al. Pregnancy intention and its relationship to birth and maternal outcomes. Obstet Gynecol 2007;109:678-86.

18. Singh S, Darroch J, Ashford L. Adding It Up: The Costs and Benefits of Investing in Sexual and Reproductive Health 2014. New York: Guttmacher Institute, 2014.

19. Ulikpan A, Mirzoev T, Jimenez E, et al. Central Asian Post-Soviet health systems in transition: has different aid engagement produced different outcomes? Glob Health Action 2014;7:24978.

20. Health care in Central Asia. Buckingham: Open University Press, 2002.

21. Tajikistan Demographic and Health Survey 2012. Dushanbe, Tajikistan, and Calverton, Maryland, USA: SA, MOH, and ICF International. Statistical Agency under the President of the Republic of Tajikistan (SA), Ministry of Health [Tajikistan], and ICF International 2013.

22. Cleland J, Ali MM. Reproductive consequences of contraceptive failure in 19 developing countries. Obstet Gynecol 2004;104:314-20.

23. Trussell J. Contraceptive efficacy. In: Hatcher R, Trussell J, Nelson A eds. Contraceptive Technology: Twentieth Revised Edition. 20th ed. New York: NY: Ardent Media, 2011.

24. Trussell J. Contraceptive efficacy: Global library of women's medicine. 2014. Available from. http://www.glowm.com/section_ view/heading/Contraceptive\%20Efficacy/item/374.

25. World Health Organization. Medical eligibility criteria for contraceptive use. 5th edn, 2015.
26. Sedgh G, Ashford L, Hussain R. Unmet Need for Contraception in Developing Countries: Examining Women's Reasons for Not Using a Method. New York: Guttmacher Institute, 2016.

27. Free C, Knight R, Robertson S, et al. Smoking cessation support delivered via mobile phone text messaging (txt2stop): a single-blind, randomised trial. Lancet 2011;378:49-55.

28. Lester RT, Ritvo P, Mills EJ, et al. Effects of a mobile phone short message service on antiretroviral treatment adherence in Kenya (WelTel Kenya1): a randomised trial. Lancet 2010;376:1838-45.

29. Pop-Eleches $\mathrm{C}$, Thirumurthy $\mathrm{H}$, Habyarimana JP, et al. Mobile phone technologies improve adherence to antiretroviral treatment in a resource-limited setting: a randomized controlled trial of text message reminders. AIDS 2011;25:825-34

30. Zurovac D, Sudoi RK, Akhwale WS, et al. The effect of mobile phone text-message reminders on Kenyan health workers' adherence to malaria treatment guidelines: a cluster randomised trial. Lancet 2011;378:795-803

31. Orr JA, King RJ. Mobile phone SMS messages can enhance healthy behaviour: a meta-analysis of randomised controlled trials. Health Psychol Rev 2015;9:397-416.

32. Hall CS, Fottrell E, Wilkinson S, et al. Assessing the impact of mHealth interventions in low- and middle-income countries - what has been shown to work? Glob Health Action 2014;7:25606.

33. Aranda-Jan CB, Mohutsiwa-Dibe N, Loukanova S. Systematic review on what works, what does not work and why of implementation of mobile health (mHealth) projects in Africa. BMC Public Health 2014;14:188.

34. Free C, Phillips G, Galli L, et al. The effectiveness of mobile-health technology-based health behaviour change or disease management interventions for health care consumers: a systematic review. PLoS Med 2013;10:e1001362

35. Head KJ, Noar SM, lannarino NT, et al. Efficacy of text messagingbased interventions for health promotion: a meta-analysis. Soc Sci Med 2013;97:41-8.

36. Hall AK, Cole-Lewis H, Bernhardt JM. Mobile text messaging for health: a systematic review of reviews. Annu Rev Public Health 2015;36:393-415.

37. L'Engle KL, Mangone ER, Parcesepe AM, et al. Mobile phone interventions for adolescent sexual and reproductive health: a systematic review. Pediatrics 2016;138:e20160884.

38. Berenson AB, Rahman M. A randomized controlled study of two educational interventions on adherence with oral contraceptives and condoms. Contraception 2012;86:716-24.

39. Castaño PM, Bynum JY, Andrés R, et al. Effect of daily text messages on oral contraceptive continuation: a randomized controlled trial. Obstet Gynecol 2012;119:14-20.

40. Trent M, Thompson C, Tomaszewski K. Text Messaging Support for Urban Adolescents and Young Adults Using Injectable Contraception: Outcomes of the DepoText Pilot Trial. J Adolesc Health 2015:57:100-6.

41. Hall KS, Westhoff CL, Castaño PM. The impact of an educational text message intervention on young urban women's knowledge of oral contraception. Contraception 2013;87:449-54.

42. Smith C, Gold J, Ngo TD, et al. Mobile phone-based interventions for improving contraception use. Cochrane Database Syst Rev 2015:CD011159.

43. Higgins JP, Altman DG, Gøtzsche PC, et al. The cochrane collaboration's tool for assessing risk of bias in randomised trials. BMJ 2011;343:d5928.

44. Smith C, Ngo TD, Gold J, et al. Effect of a mobile phone-based intervention on post-abortion contraception: a randomized controlled trial in Cambodia. Bull World Health Organ 2015;93:842-50.

45. Mobile cellular subscriptions per 100 people: World Bank (International Telecommunication Union, World Telecommunication/ ICT Development Report and database. 2015 http://data.worldbank. org/indicator/IT.CEL.SETS.P2 (20 Feb 2017).

46. Study on the use of mobile technologies in Tajikistan. 2015. ICT4D https://ict4d.tj/2015/04/issledovanie-po-ispolzovaniyu-mobilnyihtehnologiy-v-tadzhikistane-infographics/\#prettyPhoto.

47. Bartholomew Eldredge LK, Markham C, Ruiter R, et al. Planning Health Promotion Programs: An Intervention Mapping Approach. 4th edn, 2016.

48. Montaño D, Kasprzyk D. Theory of Reasoned Action, Theory of Planned Behavior, and the Integrated Behavioral Model. In: Glanz K, Rimer BK, Viswanath K, eds. Health behaviour: Theory, research and practice. 5th ed. San Francisco: Jossey-Bass, 2015:168-222.

49. Kok G, Gottlieb NH, Peters GJ, et al. A taxonomy of behaviour change methods: an Intervention Mapping approach. Health Psychol Rev 2016;10:297-312. 
50. Francis JJ, Eccles MP, Johnston M, et al. Constructing questionnaires based on the theory of planned behaviour. A manual for health services researchers 2010;2004:2-12.

51. Montaño DE, Kasprzyk D, Hamilton DT, et al. Evidence-based identification of key beliefs explaining adult male circumcision motivation in Zimbabwe: targets for behavior change messaging. AIDS Behav 2014;18:885-904.

52. O'Kelly M, Ratitch B. Clinical trials with missing data: A guide for practitioners. John Wiley \& Sons, Ltd, 2014.

53. Schulz KF, Altman DG, Moher D, et al. CONSORT 2010 statement: updated guidelines for reporting parallel group randomised trials. BMJ 2010;340:c332.

54. Guideline on adjustment for baseline covariates in clinical trials. European Medicines Agency CfMPfHU, 2015.
55. Pocock SJ, Assmann SE, Enos LE, et al. Subgroup analysis, covariate adjustment and baseline comparisons in clinical trial reporting: current practice and problems. Stat Med 2002;21:2917-30.

56. Kasenda B, Schandelmaier S, Sun X, et al. Subgroup analyses in randomised controlled trials: cohort study on trial protocols and journal publications. BMJ 2014;349:94539.

57. Gabler NB, Duan N, Liao D, et al. Dealing with heterogeneity of treatment effects: is the literature up to the challenge? Trials 2009;10:43

58. Kent DM, Rothwell PM, loannidis JP, et al. Assessing and reporting heterogeneity in treatment effects in clinical trials: a proposal. Trials 2010;11:85

59. Brookes ST, Whitely E, Egger M, et al. Subgroup analyses in randomized trials: risks of subgroup-specific analyses; power and sample size for the interaction test. J Clin Epidemiol 2004;57:229-36. 